\title{
THE EXISTENCE OF NONTRIANGULABLE CUT LOCI
}

\author{
BY DAVID SINGER AND HERMAN GLUCK ${ }^{1}$ \\ Communicated by S. S. Chern, March 5, 1976
}

We continue in this note the description of deformation theorems for geodesic fields on a Riemannian manifold begun in [1], restricting ourselves here to surfaces of revolution and to deformations of metric within this class. Using real and holomorphic Fourier transforms, we obtain in Theorem 2 an explicit formula for the deformation of metric corresponding to a prescribed deflection of geodesics. As an application, we turn again to the structure of the cut locus and prove

THEOREM 1. There exists in $R^{3}$ a strictly convex surface of revolution containing a nonempty open set of points $p$ for which the cut locus $C(p)$ is nontriangulable.

We thank Professors Robert Strichartz, Oscar Rothaus and Emil Grosswald for many helpful conversations.

1. Geodesics on a surface of revolution. Let $M$ be a surface of revolution whose metric is given in polar coordinates on the disc $r \leqslant 1$ by

$$
d s^{2}=E(r) d r^{2}+r^{2} d \theta^{2}
$$

Then the equation of a geodesic $\gamma(t)=(r(t), \theta(t))$ is given explicitly [4] by

$$
\theta=\theta_{0}+\int_{r_{0}}^{r} \frac{c \sqrt{E(r)}}{r \sqrt{r^{2}-c^{2}}} d r
$$

where the quantity $|c|$ measures the closest approach (in the $r-\theta$ plane) of the geodesic to the origin. Note that the constant $c$ can be computed from any small segment of the geodesic by Clairaut's theorem [4]:

$$
c=r(t) \sin \epsilon(t)
$$

where $\epsilon(t)$ is the angle between $\gamma$ and the meridian $\theta=$ constant.

AMS (MOS) subject classifications (1970). Primary 53C20; Secondary 42A68.

${ }^{1}$ We thank the National Science Foundation for financial support.

Copyright $\odot$ 1976, American Mathematical Society 

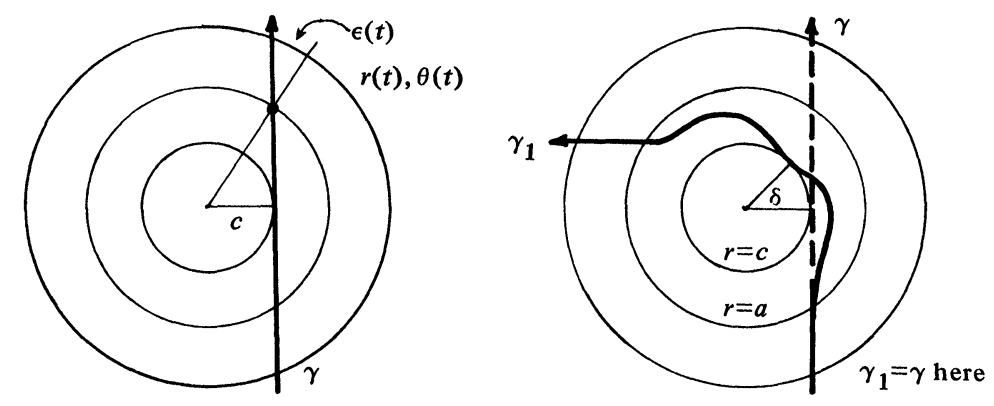

Suppose now that the metric is altered on some $\operatorname{disc} r<a<1$, but remains rotationally symmetric: $d s_{1}^{2}=E_{1}(r) d r^{2}+r^{2} d \theta^{2}$. Let $\gamma$ be a geodesic in the original metric and $\gamma_{1}$ the geodesic in the perturbed metric which begins outside the disc $r<a$ coincident with $\gamma$. By (2), both geodesics correspond to the same value of $c$. So if $\gamma$ is tangent to the circle $r=c$ at the point $(c, \theta)$ and $\gamma_{1}$ at the point $\left(c, \theta_{1}\right)$, then the angle $\delta(c)=\theta_{1}-\theta$ measures the deflection of $\gamma$ due to the change of metric. Subtracting (1) from the corresponding formula in the new metric, we get

$$
\delta(c)=\int_{c}^{1} \frac{c g(r)}{r \sqrt{r^{2}-c^{2}}} d r,
$$

where $g(r)=\sqrt{E_{1}(r)}-\sqrt{E(r)}$ measures the change in metric.

Conversely, we prove

THEOREM 2. Given a preassigned deflection function $\delta$, there exists a corresponding change of metric which realizes it.

Specifically, let $\delta$ be a smooth odd function supported on $[-a, a]$. Then there is a smooth even function $g$, vanishing at 0 and also supported on $[-a, a]$, such that $\delta$ and $g$ are related by equation (3). Indeed, $g$ is given explicitly by

$$
g(r)=\frac{2}{\pi} J_{r}^{\infty} \frac{\left[\delta(c)-c \delta^{\prime}(c)\right] r^{2}}{c^{2} \sqrt{c^{2}-r^{2}}} d c .
$$

2. Proof of Theorem 2 . We briefly sketch the steps involved.

Step 1. Changing variables by $r=e^{-x}$ and $c=e^{-v}$, (3) becomes

$$
H(v)=\int_{-\infty}^{\infty} G(x) K(v-x) \frac{d x}{\sqrt{2 \pi}}=G * K \quad \text { (convolution), }
$$

where $H(v)=\delta\left(e^{-v}\right), G(x)=g\left(e^{-x}\right)$ and the kernel $K$ is defined by

$$
K(u)= \begin{cases}\sqrt{2 \pi}\left(e^{2 u}-1\right)^{-1 / 2} & \text { for } u>0 \\ 0 & \text { for } u \leqslant 0\end{cases}
$$


Step 2. Applying the Fourier transform $T$ to (5), we get

$$
T(H)=T(G) T(K)
$$

The transform of $K$ turns out to be a Beta function:

$$
T(K)(t)=\frac{1}{2} \frac{\Gamma(1 / 2) \Gamma(1 / 2+i t / 2)}{\Gamma(1+i t / 2)}=\frac{1}{2} B(1 / 2,1 / 2+i t / 2) .
$$

This is nonvanishing, so equation (7) can be solved for $T(G)$ :

$$
T(G)=T(H) / T(K) .
$$

Step 3. Given the deflection function $\delta$, and hence $H$, we show that $T(H) / T(K)$ lies in the Schwarz space of smooth functions which decay "rapidly" at $\pm \infty$. Since this is in the image of Fourier transform, we can solve equation (8) for $G$.

Step 4. Finally we "de-exponentiate" $G$ to retrieve the function $g$. Using either the Paley-Wiener theorem for holomorphic Fourier transforms or a direct argument from equation (4), we show that $g$ satisfies the conditions listed in Theorem 2, completing the proof.

3. Proof of Theorem 1. The geodesics from a point $P$ on the equator of the unit sphere in $R^{3}$ converge at the antipodal point $Q$. Suppose that by perturbing the metric slightly near the north pole, we can deflect the geodesics from $P$ so that:

(a) $Q$ is still a conjugate point to $P$ along some geodesics of length $\pi$.

(b) The geodesics from $P$ which pass through $Q$ have lengths $\geqslant \pi$.

(c) There is an infinite sequence of geodesics from $P$ to $Q$ of length $\pi$, inter leaved with an infinite sequence of lengths $>\pi$.

From (a) and (b) it will follow that $Q$ is still in the cut locus of $P$. Furthermore, on each geodesic from $P$ to $Q$ of length $>\pi$, the cut point will occur before $Q$. By (c), removal of $Q$ will disconnect the cut locus into infinitely many components, showing it to be nontriangulable.

To achieve this situation, we first describe a deflection function which can force the above conditions and then, applying Theorem 2 , realize it by a suitable perturbation of metric.

To complete the proof of Theorem 1, we look at any point $P$ which lies outside the region in which the metric has been changed and also outside its antipodal region. Using rotational symmetry of the new surface we obtain conditions analogous to (a), (b) and (c) above; thus the cut locus of $P$ is also nontriangulable.

\section{REFERENCES}

1. H. Gluck and D. Singer, Deformations of geodesic fields, Bull. Amer. Math. Soc. (to appear). 
2. S. Kobayashi, On conjugate and cut loci, Studies in Global Geometry and Analysis, Math. Assoc. Amer. Studies in Math., Prentice-Hall, Englewood Cliffs, N. J., 1967, pp. 96-122. MR $35 \# 3603$.

3. W. Rudin, Real and complex analysis, McGraw-Hill, New York, 1966. MR 35 \#1420.

4. D. J. Struik, Lectures on classical differential geometry, Addison-Wesley, Reading, Mass., 1950. MR 12, 127.

DEPARTMENT OF MATHEMATICS, CASE WESTERN RESERVE UNIVERSITY, CLEVELAND, OHIO 44106

DEPARTMENT OF MATHEMATICS, UNIVERSITY OF PENNSYLVANIA, PHILADELPHIA, PENNSYLVANIA 19174 